\title{
Epidermal Inclusion Cyst Following Percutaneous Trigger Finger Release: A Case Report
}

\author{
Hui-Kuang Huang ${ }^{1,2,3,4}$, Jung-Pan Wang ${ }^{1,2 *}$, Chun-Liang Tung ${ }^{5}$ and Fang-Chieh Lien ${ }^{3}$ \\ ${ }^{1}$ Department of Surgery, School of Medicine, National Yang-Ming University, Taiwan \\ ${ }^{2}$ Department of Orthopaedics and Traumatology, Taipei Veterans General Hospital, Taipei, Taiwan \\ ${ }^{3}$ Department of Orthopaedics, Chiayi Christian Hospital, Chiayi, Taiwan \\ ${ }^{4}$ Chung Hwa University of Medical Technology, Tainan, Taiwan \\ ${ }^{5}$ Department of Pathology, Chiayi Christian Hospital, Chiayi, Taiwan
}

\begin{abstract}
Epidermal inclusion cyst is a benign lesion that commonly occurs due to inclusion of epidermal cells into the dermal or deeper layers in a trauma event. Percutaneous release is a minimally invasive technique and good surgical outcomes can be achieved. However, the percutaneous procedure is a puncture injury and the epidermal inclusion cyst is reasonable to become a possible complication. In this article, we presented a case of trigger finger in left middle finger. She underwent percutaneous release as the treatment and a second percutaneous release 5 months later due to recurrence of the symptoms. An epidermal inclusion cyst was noted 5 months thereafter and it was treated with excision. With this case, we need to be more aware of this possibility if a mass lesion without infection signs occurs in the released area.
\end{abstract}

Keywords: Complication; Epidermal inclusion cyst; Percutaneous; Release; Trigger finger

\section{Introduction}

Trigger digit is a common problem encountered in clinical practice. Intrasheath injection of steroid is a good first-line treatment and good outcomes can be achieved [1]. If non-operative treatments fail, further surgical treatment should be considered. Open release and percutaneous release of the involved pulley are both commonly-used methods and both can have good surgical outcomes. Percutaneous release is a minimally invasive technique that can be performed with a puncture wound. But complications are still a concern due to the nature of the blind performance under the skin.

Epidermal inclusion cyst is a benign lesion that commonly occurs due to inclusion of epidermal cells into the dermal or deeper layers in a trauma event. The epidermal cells with growth-ability can reproduce, and produce the keratinous debris that can then form a cyst containing lamellated keratin. The tumor grows slowly, forming a round shape with the whitish color of the keratin.

\section{Case Report}

A 68-year-old woman had been well without any underlying medical disease. She visited our clinic due to recurrent trigger finger (Quinnell grade III) in the Left Middle Finger (LMF), which had been treated once with intrasheath steroid injection 5 months previous. Percutaneous release was then performed to treat her trigger finger. The surgery was performed under local anesthesia as an office-based surgery by a surgeon whose expertise was Level III (experienced specialist) in Tang's grading system and who had performed more than 1500 percutaneous release operations for trigger digits by the time of the surgery [2]. The percutaneous release was performed using an 18-gauge needle. The patient's symptoms were relieved in the postoperative 2-week follow-up. However, the symptoms recurred 4 months later, and she came back to our clinic 5 months after the surgery. The percutaneous release was performed again for the LMF. The follow-ups revealed that the triggering had been completely relieved.
However, she came back to our clinic 8 months after the second surgery and complained that a gradually growing and protruding mass had appeared at the released area 5 months after the second release. She denied any traumatic episode involving the left hand after the last operation. The mass was whitish in color just beneath the skin (Figure 1). No Kanevel signs were found at the involved LMF [3]. Aspiration was performed, but only a small amount of whitish fluid could be drawn. Painkillers and oral antibiotics were prescribed, due to a suspected diagnosis of furuncle formation. At the follow-up 1 week later, no change in the mass was seen. Aspiration was performed again, and showed the same results. No microorganism source could be identified from the microorganism culture of the first-time aspirated fluid. The patient did not want treatment for the mass, since her life was not affected by the lesion. The mass grew in size gradually and protruded more on the skin, so the patient came back to our clinic 12 months after the second aspiration (Figure 2). The protruding mass showed mild erythematous change and a central whitish color; it had increased in size from that of 1 year before. But the oval protruding mass was firm and without infection signs. Operative exploration was explained to the patient and then performed.

With a $1 \mathrm{~cm}$ oblique incision, a whitish, oval tumor with a welldemarcated and smooth margin was noted just under the skin layer. In the pathology exam, the tumor revealed a laminated content. An epidermal component with much keratin debris was noted (Figures 3 and 4). Epidermal inclusion cyst was confirmed.

*Corresponding author: Jung-Pan Wang, Department of Orthopaedics and Traumatology, Taipei Veterans General Hospital, 201, Sec 2, Shih-Pai Road, Taipei 112, Taiwan, Tel: +886-2-2875-7557; E-mail: jpwang@vghtpe.gov.tw

Received July 06, 2017; Accepted July 14, 2017; Published July 16, 2017

Citation: Huang HK, Wang JP, Tung CL, Lien FC (2017) Epidermal Inclusion Cyst Following Percutaneous Trigger Finger Release: A Case Report. J Mol Biomark Diagn 8: 357. doi: 10.4172/2155-9929.1000357

Copyright: (c) 2017 Huang HK, et al. This is an open-access article distributed under the terms of the Creative Commons Attribution License, which permits unrestricted use, distribution, and reproduction in any medium, provided the original author and source are credited. 


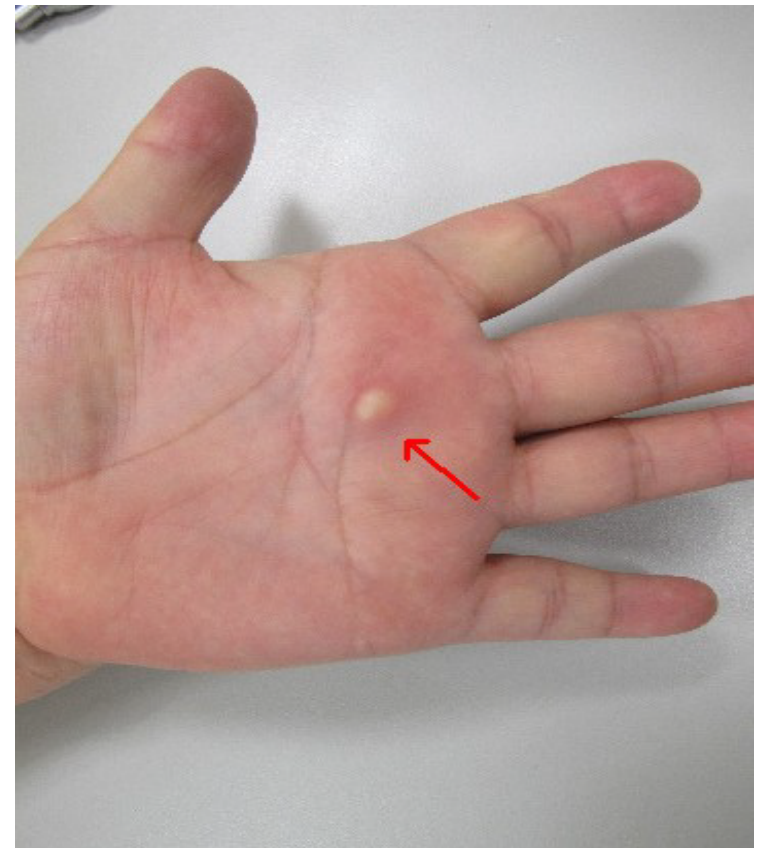

Figure 1: Photograph 8 months after the second percutaneous release, showing a protruding mass, whitish in colour, beneath the skin.

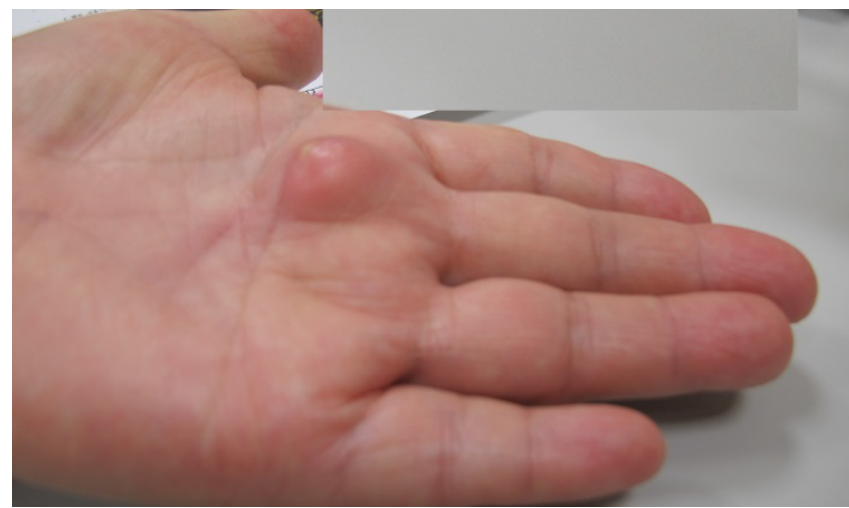

Figure 2: Photograph 20 months after the second percutaneous release, showing an enlarged mass.

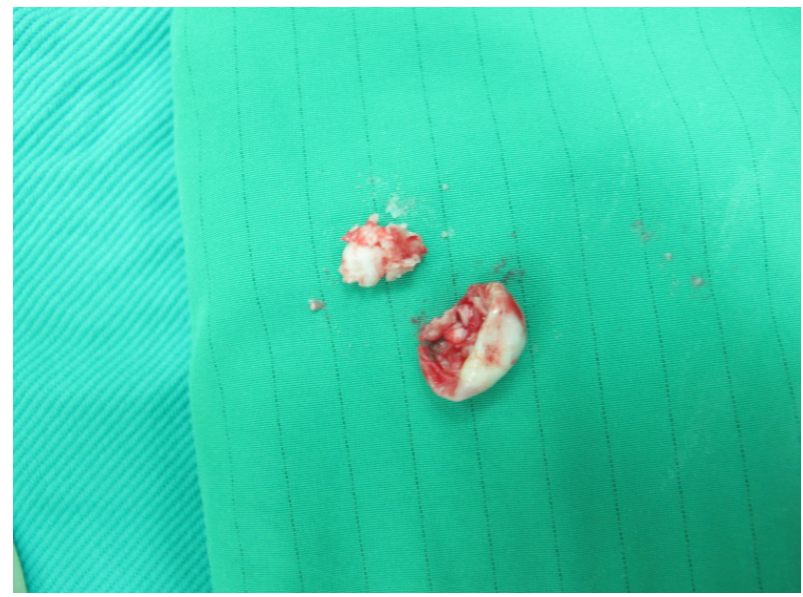

Figure 3: Content of the tumor showing whitish debris.

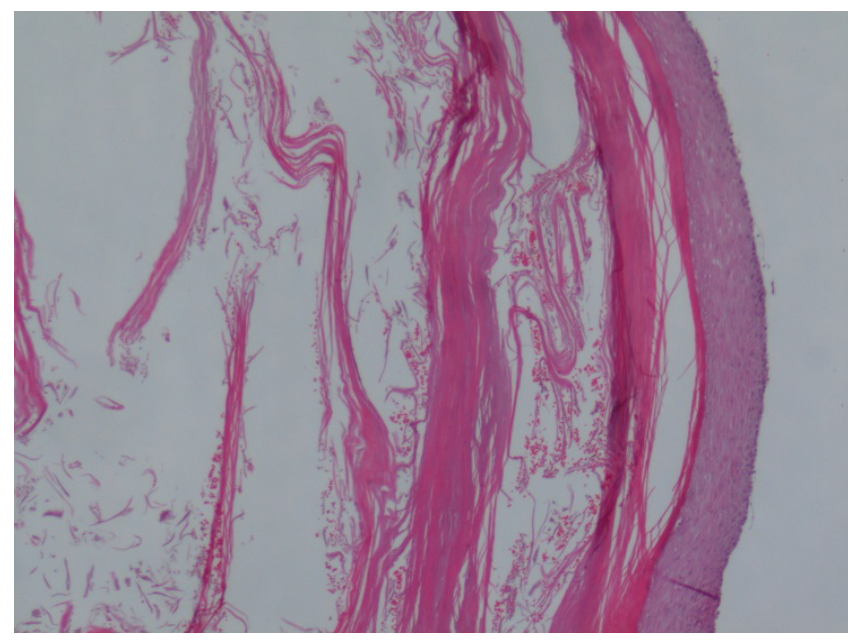

Figure 4: Photograph showing stratified squamous cell epithelial lining and eosinophilic keratin debris in the cystic cavity. (hematoxylin-eosin stain; 100x).

After excision of the cyst, the patient achieved complete resolution of the symptoms and no recurrence was seen at the 3-month follow-up.

\section{Discussion}

Percutaneous release of trigger digits was first introduced in 1985 , and the safety of the procedure and its excellent outcomes were reported [4]. But due to the proximity of the neurovascular bundles, the vulnerability of the neurovascular structures were a concern related to percutaneous release, particularly in the thumb and index finger. Of the reported complications, a tumor-like lesion arising after the percutaneous-release procedure could be infection, hematoma, scar formation, traumatic pseudoaneurysm, and traumatic neuroma [5-9]. These problems could be differentiated based on local skin presentation, duration of occurrence, and the characteristics of the mass. To our knowledge, there have been no reports of epidermal inclusion cyst formation related to the percutaneous release of trigger digit, although there was a case report of epidermal inclusion cyst formation after a bacillus Calmette-Guerin vaccination [10].

The pathogenesis of the formation of the epidermal inclusion cyst is very clear and reasonable, in that the puncture injury brings the epidermal element into the dermis layer, and then the epidermal element continues growing into the lesion. Percutaneous release is an epidermal injury, and it is reasonable that this would lead to the occurrence of the epidermal inclusion cyst, especially after repeated instances of percutaneous release. But we cannot confirm which percutaneous release or even if the previous intrasheath steroid injection caused the tumor, although only a 25 -gauge needle was used for the intra-sheath injection. In addition, other rare causes of the cyst formation could still possible.

Based on our experience with this case, we believe that the diagnosis could be made earlier. In the clinical check-up for cases with a protruding mass with central white and peripheral erythema, infection with pus accumulation should be highly suspected. If there are few infection signs, other pathogeneses should be considered. Also, cases in which no pus can be drawn out and no microbacteria can be grown in the contained fluid could indicate a diagnosis other than pyogenic accumulation. A sonographic exam, if available, could be also helpful for the diagnosis. 
Citation: Huang HK, Wang JP, Tung CL, Lien FC (2017) Epidermal Inclusion Cyst Following Percutaneous Trigger Finger Release: A Case Report. J Mol Biomark Diagn 8: 357. doi: 10.4172/2155-9929.1000357

\section{Conclusion}

In this case, we reported the appearance and pathogenesis of epidermal inclusion cyst formation, which occurred after percutaneous release of the trigger digit. In cases with a slow-growing, protruding mass after percutaneous release, surgeons should consider this possible diagnosis earlier, if other common etiologies are not likely.

\section{References}

1. Quinnell RC (1980) Conservative management of trigger finger. Practitioner 224: $187-190$.

2. Tang JB, Giddins $\mathrm{G}$ (2016) Why and how to report surgeons' levels of expertise. J Hand Surg Eur Vol 41: 365-366.

3. Kanavel A (1939) Infections of the hand. In: A guide to the surgical treatment of acute and chronic suppurative processes in the fingers, hand, and forearm. (7th edn), Lea \& Febiger, Philadelphia, PA.

4. Sato ES, Dos Santos JB, Belloti JC, Albertoni WM, Faloppa F (2014) Percutaneous release of trigger fingers. Hand Clinics 30: 39-45.
5. Samuel AT, Daniel AO, Jain S, Andrew JW (2012) Digital artery pseudoaneurysm following percutaneous trigger thumb release: a case report. J Bone Joint Surg Am 94: e6.

6. Sreedharan S, Teoh LC, Chew WY (2011) Neuroma of the radial digital nerve of the middle finger following trigger release. Hand Surg 16: 95-97.

7. Foo TL (2011) Caution against percutaneous trigger release in patients on anticoagulant. J Hand Surg Am 36: 1566-1567.

8. Zhao JG, Kan SL, Zhao L, Wang ZL, Long L, et al. ( 2014) Percutaneous first annular pulley release for trigger digits: A systematic review and meta-analysis of current evidence. J Hand Surg Am 39: 2192-2202.

9. Jongjirasiri $Y(2007)$ The results of percutaneous release of trigger digits by using full handle knife 15 degrees: An anatomical hand surface landmark and clinical study. J Med Assoc Thai 90: 1348-1355.

10. Schumacher HH, Ahmad T (2005) Epidermal inclusion cyst after bacillus calmette-guerin vaccination. Plast Reconstr Surg 115: 1449-1450. 